\title{
Monitoring of eye lens doses in radiation protection
}

\author{
J.-M. Bordy \\ CEA, LIST, Laboratoire National Henri Becquerel (LNE LNHB), 91191 Gif sur Yvette Cedex, France.
}

Received 29 December 2014 - Accepted 20 May 2015

\begin{abstract}
Mainly due to the ICRP recommendation to decrease the exposure limit for eye lenses, the eye lens dosimetry has to be reconsidered. This paper gives an overview of the issues raised after this recommendation; that is to say, the choice and definition of the operational quantity to be monitored, the type testing and calibration of dosimeters aimed at measuring eyes lens "doses", the design of existing eye lens dosimeters and their wearing conditions. Finally, a criterion to choose between a direct measurement of the personal dose equivalent at three millimeters depth, $H_{\mathrm{p}}(3)$, with a dedicated dosimeter, and an indirect evaluation of $H_{\mathrm{p}}(3)$ through whole-body monitoring is presented.
\end{abstract}

Keywords: eye lens / dosimetry / radiation protection

\section{Introduction}

Over the past few years, many articles, oral communications and/or posters during conferences have dealt with eye lens dosimetry. The two main reasons for this keen interest were the results of epidemiological studies, for instance for workers in the medical sector, which showed the existence of cases of cataracts for doses lower than 0.5 Gy (Nakashima et al., 2006; Worgul et al., 2007; Chodick et al., 2008), and subsequently the recommendation by the ICRP to decrease the exposure limit for workers from $150 \mathrm{mSv}$ per year to $20 \mathrm{mSv}$ in a year averaged over defined periods of five consecutive years, with no single year exceeding $50 \mathrm{mSv}$ (ICRP, 2011). These recommendations are introduced in the European directive 2013/59 (EURATOM, 2013).

Figure 1 summarizes the method which allows the limit of occupational exposure to be controlled in terms of the equivalent dose $H_{\mathrm{T}}$ and effective dose $E$, which are the protection quantities defined to ensure that the occurrence of stochastic health effects is kept below unacceptable levels and that tissue reactions are avoided. "Q" is the quality factor for charged particles; it is applied to absorbed energy calculations in simplified phantoms (a slab for the trunk, cylinders for the finger, wrist, ankle and head). The head phantom was introduced in ISO 29661 (ISO, 2012); the update of the ISO 4037 (ISO, 1999) series started in 2015. " $\mathrm{w}_{R}$ " and " $\mathrm{w}_{\mathrm{T}}$ " are the weighting factors for radiation and tissue, respectively, used to calculate protection quantities in anthropomorphic phantoms. An operational quantity is used to estimate a protection quantity, under the assumption that the shape of the phantoms and the

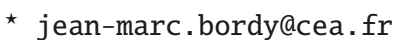

weighting and quality factors together will lead to similar values of operational and protection quantities for given radiation fields ${ }^{1}$. The dosimeters are designed according to the characteristics of the operational quantities to be measured (angle and energy response). They are tested according to criteria which can be found in IEC and ISO standards such as IEC 62387 (IEC, 2012), 60846 (IEC, 2007), 61066 (IEC, 2006) and 61526 (IEC, 2005), and ISO 12794 (ISO, 2000). The dosimeter is then calibrated in terms of the operational quantity in conditions representative of normal everyday use. For the dosimetry of the eye lens, the exposure limit is expressed in terms of the equivalent dose to the eye lens, $H_{\text {lens }}$; the dedicated operational quantities are the personal dose equivalent $H_{\mathrm{p}}(3)$ and directional dose equivalent $H^{\prime}(3)$. Today, it is unanimously agreed that eye lens doses must be measured, but the modalities of its implementation are the cause of much debate.

It is necessary to briefly recall that the three main characteristics of a radiation field are, on one hand, its spatial and temporal homogeneity, and on the other hand, the exposure level in terms of the dose equivalent, which may or may not be close to the exposure limit.

In this paper, we deal with the choice of the operational quantity from the point of view of metrology and the routine measurement methods, either for a direct measurement, or an indirect evaluation of the eye lens "doses". The implementation of occupational dosimetry through normal everyday use and the design of an individual dosimeter are also discussed. A method is proposed to define a threshold in terms of the "dose" below which it is not necessary to make a direct measurement of $H_{\mathrm{p}}(3)$. Some of the options described in this paper

\footnotetext{
1 Under the condition that operational quantities will slightly overestimate protection quantities.
} 


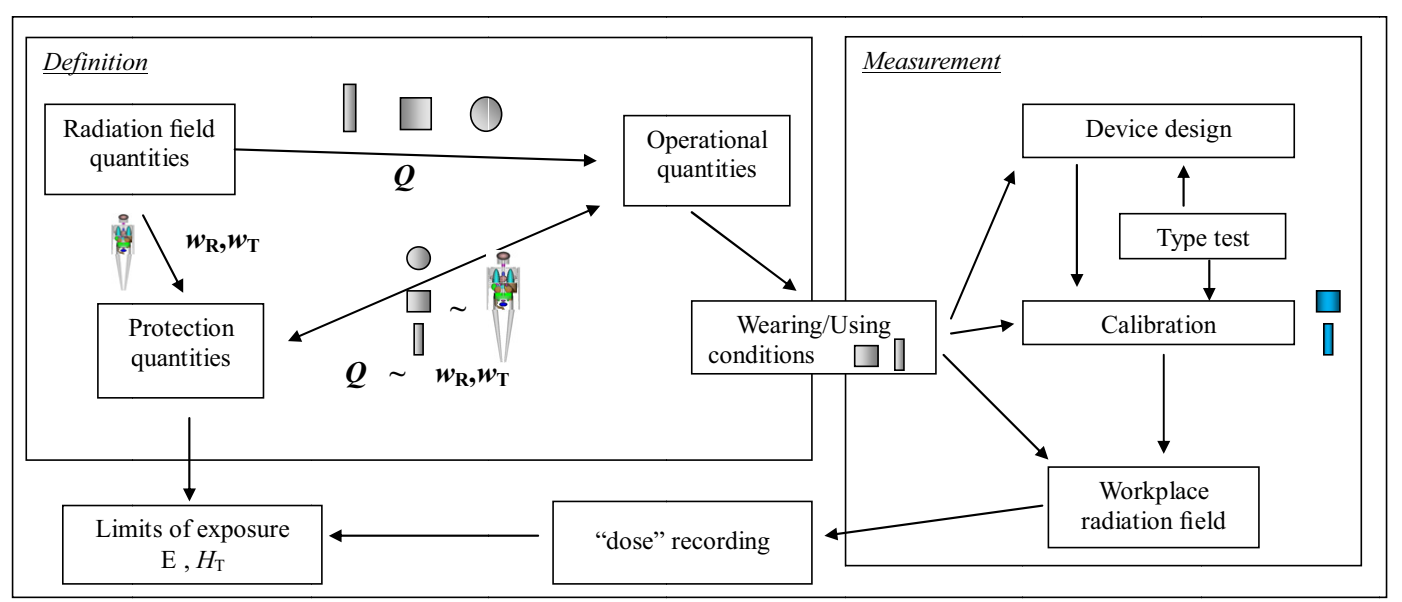

Figure 1. Synopsis of the method applied to the radiation protection dosimetry.

have been partially taken from standards; however, in certain cases, the inferences are different.

\section{Choice of the operational quantity to monitor eye lens exposures}

First of all, the personal dose equivalent at $3 \mathrm{~mm}$ depth, $H_{\mathrm{p}}(3)$, was defined in ICRU Reports 43 and 47 (ICRU, 1988, 1992) but no conversion coefficient ${ }^{2}, h_{\mathrm{p}}(3)$, was officially agreed. One of the tasks of ENEA and CEA LIST LNHB within ORAMED was to propose a new definition of the personal dose equivalent $H_{\mathrm{p}}(3)$ (Daures et al., 2011; Gualdrini et al., 2011). For this definition, a new phantom was proposed, as had previously been done for the finger and the wrist. This new cylindrical phantom is made of tissue-equivalent ICRU material and has a height and diameter equal to $20 \mathrm{~cm}$. It was chosen because of its simple shape and size, which are close to the dimensions of an adult head. Conversion coefficients $h_{\mathrm{p}}(3)$ were calculated (Daures et al., 2009; Mariotti et al. 2009; Gualdrini et al., 2013) through Monte Carlo calculations using PENELOPE (Salvat et al., 2006) and MCNP (Pelowitz, 2005). All values of $H_{\mathrm{p}}(3)$ used in this paper are those corresponding to this cylindrical phantom. Note that this phantom is also used for control in dental radiology.

As the operational quantities are used as estimators of the protection quantities, in order to choose the most suitable quantity to estimate $H_{\text {lens }}$, we compare the operational quantities for the whole body, $H_{\mathrm{p}}(10)$ and $H^{*}(10)$, the extremities, $H_{\mathrm{p}}(0.07)$ and $H^{\prime}(0.07)$, and the eye lens, $H^{\prime}(3)$ and $H_{\mathrm{p}}(3)$, defined in a cylinder $20 \mathrm{~cm}$ in diameter, with $H_{\text {lens }}$. More precisely, we compare the conversion coefficients, $h$, from air kerma toward the operational and protection quantities.

As an example, Figure 2 shows the comparison of three dose equivalent quantities at $0^{\circ}$ incidence (ICRU 57, 1995; Daures et al., 2009) with the absorbed dose to the eye lens for anterior-posterior irradiation (ICRP 74, 1995). It should be

${ }^{2}$ Small " $h$ " denotes the conversion coefficients here from air kerma to the dose equivalent at $3 \mathrm{~mm}$ depth $h_{\mathrm{p}}(3)=H_{\mathrm{p}}(3) / K \mathrm{a}$. noted that all these calculations were made with the kerma approximation assumption; that is to say, secondary charged particles are not transported, which is not representative of the energy deposition in matter when electronic equilibrium is not fulfilled. From this comparison, the personal dose equivalents $H_{\mathrm{p}, \mathrm{KA}}\left(3,0^{\circ}\right)$, calculated with the kerma approximation ${ }^{3}$, and $H_{\mathrm{p}}\left(0.07,0^{\circ}\right)$, stand out as the best solution from a metrological point of view because of their similarity to the equivalent

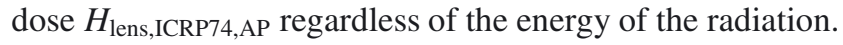

Until now, the conversion coefficients for operational quantities and protection quantities have been calculated using the kerma approximation (ICRP, 1995; ICRU, 1995). That is to say, it is considered that the energy is absorbed in the vicinity of the interaction. The values of protection quantities are now calculated without the kerma approximation (ICRP 116, 2010), taking into account the follow-up of the secondary particles produced after interaction of the photons. Thus, in the future, new sets of conversion coefficients for the operational quantity will be calculated in the same conditions (Daures et al., 2009, 2011). One can see in Figure 3 that the eye lens dose equivalent and equivalent dose decrease to below one $\mathrm{MeV}$ due to the lack of electronic equilibrium when secondaries are followed. The same decrease exists for the dose equivalent at 0.07 and $10 \mathrm{~mm}$ depth but for energy of about $700 \mathrm{keV}$ and three $\mathrm{MeV}$, respectively. Thus, this will lead to a larger discrepancy between the operational quantities defined at the depths $0.07,3$ and $10 \mathrm{~mm}$, at least for the energies higher than approximately $700 \mathrm{keV}$ compared with the former situation described in Figure 2. Of course, all the conversion coefficients are calculated in a vacuum; therefore, they do not take into account the electrons generated in air around the phantom, so taking into account these electrons the decrease would be less abrupt. Nevertheless, it strengthens the conclusion that using $H_{\mathrm{p}, \mathrm{FT}}(3)$, calculated without kerma approximation ${ }^{4}$ in the cylindrical phantom, is the best choice for monitoring eye lens doses for its similarity to the equivalent dose $H_{\text {lens,ICRP116,AP }}$ regardless of the energy or angle of incidence of the radiation (Figure 3).

\footnotetext{
3 "KA" means "kerma approximation".

4 "FT" means "full transport", no kerma approximation.
} 


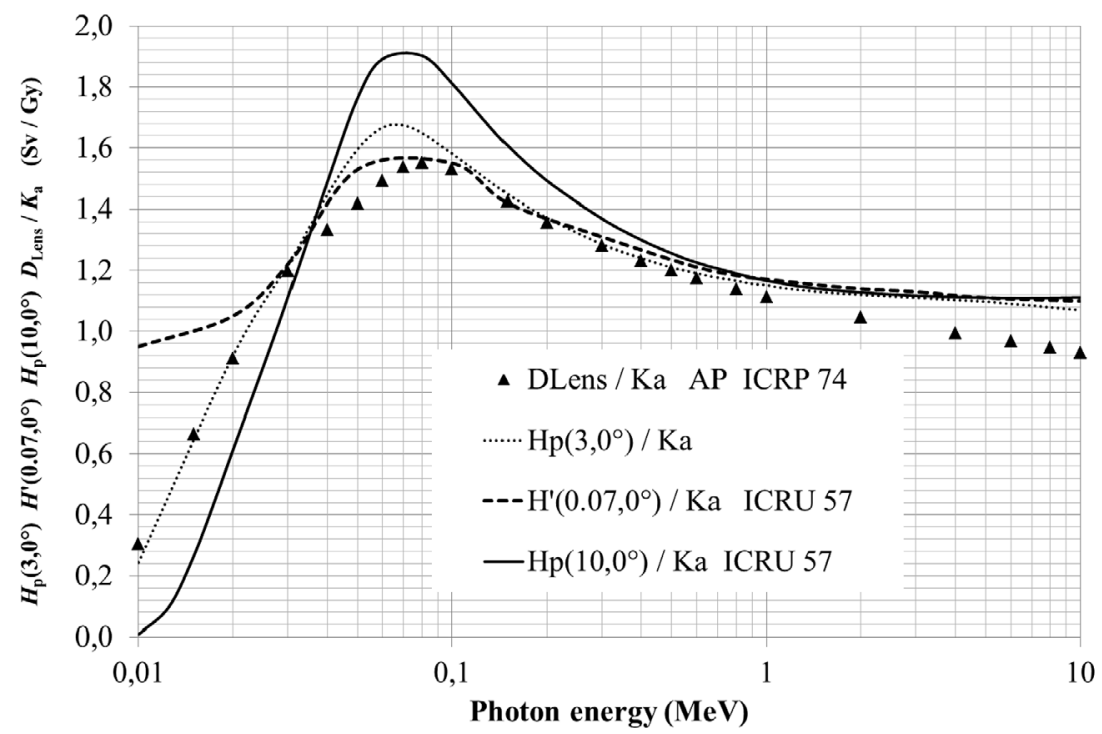

Figure 2. Comparison of dose equivalents at 10 and $0.07 \mathrm{~mm}$ depth at $0^{\circ}$ taken from the ICRU 57 report and the dose equivalent at $3 \mathrm{~mm}$ depth taken from Daures et al. (2009) with the absorbed dose to the eye lens in the anthropomorphic phantom for anterior-posterior (AP) irradiation taken from ICRP 74. $h^{\prime}(0.07)$ was plotted instead of $h_{\mathrm{p}}(0.07)$ because $h_{\mathrm{p}}(0.07)$ is not given above one MeV in ICRU 57 , but is very similar to $h^{\prime}(0.07)$ below one $\mathrm{MeV}$.

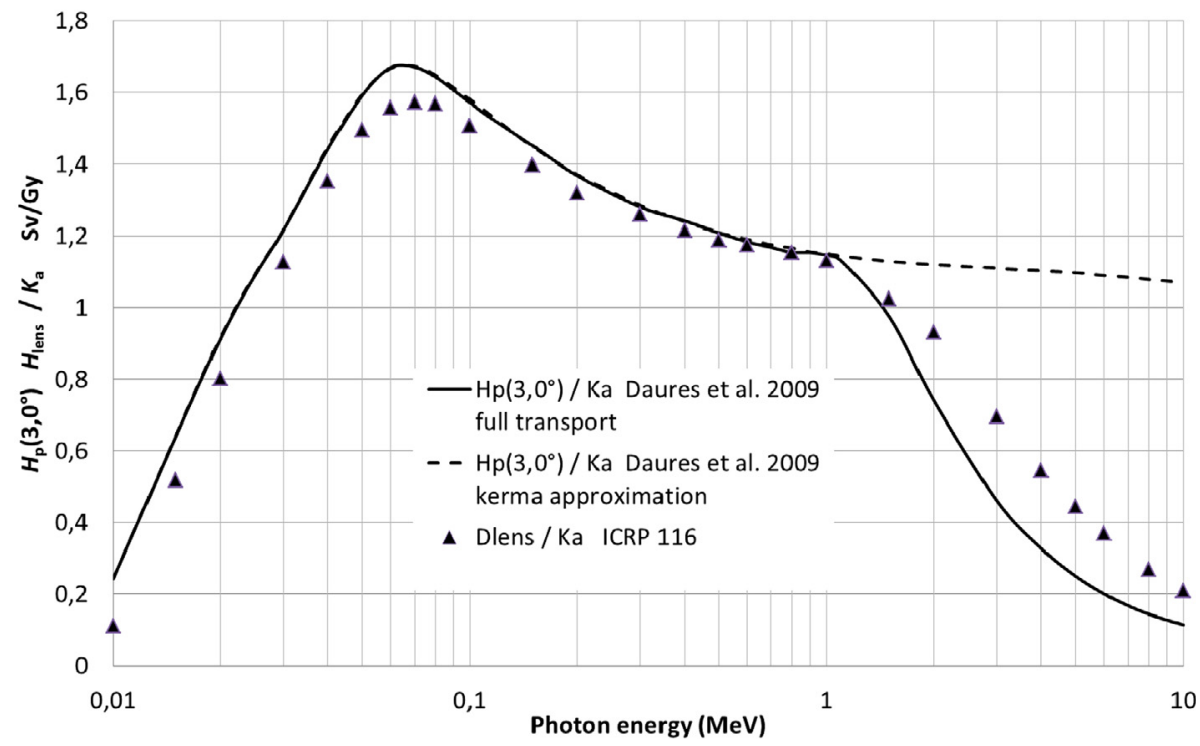

Figure 3. Comparison of dose equivalents at $3 \mathrm{~mm}$ depth at $0^{\circ}$ taken from Daures et al. (2009) calculated with full transport and kerma approximation with the equivalent dose to the eye lens in the anthropomorphic phantom for anterior-posterior irradiation derived from ICRP 116.

It is useful to underline that using a quantity which exhibits larger discrepancies with $H_{\text {lens }}$ than $H_{\mathrm{p}}(3)$, defined in the cylindrical phantom, introduces a systematic error. Tables 1 and 2 show the magnitude of this error in the case of some standard radiation fields taken in ISO 4037 (ISO, 1999) and a radiation field obtained from a medical accelerator at the LNHB with an average energy in terms of fluence equal to $6.3 \mathrm{MeV}$ (Dusciac et al., 2013) denoted LNHB LINAC; the results are normalized to $D_{\text {lens }}$ of ICRP 116 (ICRP, 2010) for AP irradiation in Table 1 and lateral (Lat) irradiation in Table 2, denoted $D_{\text {lens,ICRP,116,AP }}$ and $D_{\text {lens,ICRP,116,Lat }}$, respectively. In these tables, note that the first column gives the absorbed dose to the lens of the eye based on ICRU 74 and calculated with the kerma approximation. Also, the systematic error is only due to the use of the quantity; it does not take into account the other systematic errors due to the calibration conditions of the dosimeter or the non-homogeneity of the radiation field between the different locations of the dosimeter in normal use (trunk, finger, wrist, ankle and head).

At $0^{\circ}$ (Table 1 ), for energies below about $1 \mathrm{MeV}\left({ }^{60} \mathrm{Co}\right)$, $D_{\text {lens,ICRP74,AP }}$ is similar to $D_{\text {lens,ICRP116,AP }}+/-3 \%$ even if the quantity values were calculated with and without kerma approximation, respectively. Indeed, a depth of three millimeters is thick enough to reach electronic equilibrium below one $\mathrm{MeV}$. Looking at the operational quantities, for energies lower than about one $\mathrm{MeV}$, only $H_{\mathrm{p}}\left(10,0^{\circ}\right)$ is significantly 
Table 1. Comparison of the quantities for various radiation fields, normalized to $D_{\text {lens,ICRP,116,AP. }}$.

\begin{tabular}{|c|c|c|c|c|c|}
\hline $\begin{array}{l}\text { Radiation } \\
\text { quality }\end{array}$ & $D_{\text {lens,ICRP74,AP }}$ & $H_{\mathrm{p}, \mathrm{KA}}\left(3,0^{\circ}\right)$ & $H_{\mathrm{p}, \mathrm{FT}}\left(3,0^{\circ}\right)$ & $\begin{array}{c}\boldsymbol{H}_{\mathrm{p}}\left(\mathbf{0 . 0 7 , 0 ^ { \circ } )}\right. \\
\text { ICRU } 57\end{array}$ & $\begin{array}{c}H_{\mathrm{p}}\left(10,0^{\circ}\right) \\
\text { ICRU } 57\end{array}$ \\
\hline W80 & 0.97 & 1.07 & 1.07 & 1.03 & 1.18 \\
\hline W150 & 1.01 & 1.05 & 1.04 & 1.02 & 1.19 \\
\hline W300 & 1.02 & 1.03 & 1.03 & 1.04 & 1.12 \\
\hline $\begin{array}{l}{ }^{137} \mathrm{Cs} \\
(0.662 \mathrm{MeV})\end{array}$ & 0.99 & 1.01 & 1.01 & 1.03 & 1.04 \\
\hline $\begin{array}{l}{ }^{60} \mathrm{Co} \\
(1.25 \mathrm{MeV})\end{array}$ & 1.02 & 1.06 & 1.02 & $1.08^{*}$ & 1.07 \\
\hline LNHB LINAC & 2.17 & 2.43 & 0.65 & $2.28^{*}$ & 2.61 \\
\hline
\end{tabular}

* These are the values of $H^{\prime}(0.07)$ because $H_{\mathrm{p}}(0.07)$ is not given above one $\mathrm{MeV}$ in ICRU 57 , but are very similar due to the shape of the spherical and cylindrical phantoms.

Table 2. Comparison of the quantities for various radiation fields, normalized to $D_{\text {lens,ICRP,116,Lat }}$.

\begin{tabular}{|c|c|c|c|c|c|c|c|}
\hline $\begin{array}{l}\text { Radiation } \\
\text { quality }\end{array}$ & $D_{\text {lens,ICRP74,Lat }}$ & $H_{\mathrm{p}, \mathrm{KA}}\left(3,90^{\circ}\right)$ & $H_{\mathrm{p}, \mathrm{FT}}\left(3,90^{\circ}\right)$ & $\begin{array}{c}H p\left(0.07,75^{\circ}\right) \\
* * \text { ICRU } 57\end{array}$ & $\begin{array}{c}H^{\prime}\left(0.07,90^{\circ}\right) \\
\text { ICRU } 57\end{array}$ & $\begin{array}{c}H_{\mathrm{p}}\left(10,75^{\circ}\right) \\
* * \text { ICRU } 57\end{array}$ & $\begin{array}{c}H^{\prime}\left(10,90^{\circ}\right) \\
\text { ICRU } 57\end{array}$ \\
\hline W80 & 0.87 & 1.03 & 1.03 & 1.37 & 1.05 & 0.95 & 0.62 \\
\hline W150 & 0.94 & 1.06 & 1.06 & 1.33 & 1.09 & 1.00 & 0.72 \\
\hline W300 & 0.96 & 1.07 & 1.08 & 1.32 & 1.14 & 1.03 & 0.81 \\
\hline${ }^{137} \mathrm{Cs}$ & 0.98 & 1.07 & 1.07 & 1.24 & 1.03 & 1.03 & 0.88 \\
\hline${ }^{60} \mathrm{Co}$ & 0.97 & 1.06 & 1.07 & - & 1.01 & 1.03 & 0.92 \\
\hline LNHB LINAC & 1.20 & 1.25 & 01.01 & - & 1.21 & 1.29 & 1.15 \\
\hline
\end{tabular}

** $H_{\mathrm{p}}\left(10,75^{\circ}\right)$ and $H_{\mathrm{p}}\left(0.07,75^{\circ}\right)$ values are given only as examples because $H_{\mathrm{p}}(10)$ and $H_{\mathrm{p}}(0.07)$ do not exist in the slab phantom for angles beyond $75^{\circ}$ in ICRU 57.

different but still overestimating the protection quantity, up to $19 \%$, to $D_{\text {lens,ICRP1 } 16, A P}$.

On the contrary, a huge overestimate of $D_{\text {lens,ICRP116,AP }}$ by

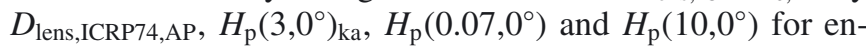
ergies above one $\mathrm{MeV}$ is observed. This is due to the lack of electronic equilibrium which forbids the use of kerma approximation beyond one $\mathrm{MeV}$, as can be seen in Figure 3. It should be noted that, although the calculation method is the same, that is to say without kerma approximation, $H_{\mathrm{p}}\left(3,0^{\circ}\right)$ underestimate of $35 \% \mathrm{D}_{\text {lens,ICRP116,AP, }}$ it can be explained by the shape of the phantom; indeed, although a cylinder is better than a cube to represent the head, the anthropomorphic head phantom is obviously more complex, including bones, eyes, brain, etc.

At $90^{\circ}$, the situation is slightly different. First, the quantities defined in the slab phantom, namely $H_{\mathrm{p}}(10)$ and $H_{\mathrm{p}}(0.07)$, cannot be used because this phantom cannot be used for large-angle irradiation. Second, the overestimate for high energies above one $\mathrm{MeV}$ of $D_{\text {lens,ICRP116,Lat }}$ is smaller than that for $D_{\text {lens,ICRP1 } 16, \mathrm{AP}}$ and $H_{\mathrm{p}}\left(3,90^{\circ}\right)$ does not underestimate $D_{\text {lens,ICRP116,Lat }}$.

The following conclusions can be drawn:

- The dose equivalent $H_{\mathrm{p}}(3)$ defined in the cylindrical phantom and calculated without kerma approximation is similar to the equivalent dose $H_{\text {lens,ICRP116 }}$ regardless of the energy or the angle of incidence of the radiation, because of the similarity of the forms of the phantoms used to calculate the values of the conversion coefficients. The same conclusion would be drawn for the directional dose equivalent $H^{\prime}(3)$, which is very similar to the personal dose equivalent $H_{\mathrm{p}}(3)$ defined in the cylindrical phantom regardless of the angle of incidence or the energy of the radiation because of the similarity of the diameter of the cylinder $(20 \mathrm{~cm})$ and the ICRU sphere $(30 \mathrm{~cm})$.

- The dose equivalents $H_{\mathrm{p}}(0.07)$ and $H^{\prime}(0.07)$ are close to the equivalent dose $H_{\text {lens,ICRP74 }}$ except below approximately $20 \mathrm{keV}$. One recalls that these quantities can be used only for weakly penetrating radiation; so, in ISO standards (ISO, 1999), there is no value of the conversion coefficient beyond $300 \mathrm{keV}$ and in ICRU 57 (ICRU, 1995) there is no value of the conversion coefficient beyond one $\mathrm{MeV}$ for $H_{\mathrm{p}}(0.07)$.

- In every case (energy and angle), the dose equivalents $H_{\mathrm{p}}(10)$ are worse estimators of the equivalent dose $H_{\text {lens }}$ than the other operational quantities. The same conclusion would be drawn for $H^{*}(10)$. It should be mentioned that $H_{\mathrm{p}}(10)$ is not defined for angles larger than $75^{\circ}$ and that $H^{*}(10)$ is only for ambient dosimetry as $H^{\prime}(10)$ is not used even for ambient dosimetry.

Therefore, the best choice to monitor $H_{\text {lens,ICRP116 }}$ is $H_{\mathrm{p}, \mathrm{FT}}(3)$ regardless of the energy and angle of incidence.

\section{Conditions of calibration and type testing of a dosimeter to measure $H_{p}(3)$}

The study of the characteristics of the operational quantities leads to the choice of the cylindrical phantom $20 \mathrm{~cm}$ in diameter made of ICRU tissue to define the personal dose equivalent $H_{\mathrm{p}}(3)$. It is obvious that for the type testing of personal dosimeters for the eye lens, a water phantom with the 
same dimensions as those used for the definition of the quantity has to be used. Apart from this metrological reasoning, it is important to mention that the cylindrical shape of the water phantom allows dosimeters to be irradiated at all angles in one irradiation and despite the fact that it is very easy to build, this phantom is commercially available ${ }^{5}$. For calibration purposes, as is the case for extremity dosimeters with the wrist and finger phantoms, the dosimeter for the eye lens has to be put on the surface of the $20-\mathrm{cm}$-diameter water phantom. However, since calibrations are generally done at an angle of normal incidence, a water slab phantom $30 \times 30 \times 15 \mathrm{~cm}^{3}$, as for the calibration of dosimeters for the whole body, can be used because of the similarity of backscatter to the cylindrical phantom at normal incidence. The standard ISO 29661 (ISO, 2012) opens up the possibility of using both phantoms for calibration in agreement with the customer. Note, however, that to conform to the definition of $H_{\mathrm{p}}(3)$ in the cylindrical phantom a correction factor must be applied if the slab phantom is used, and that for angles larger than $75^{\circ}$ the slab phantom cannot be used due to the edges.

The type testing can be done following the specification of the following standards: IEC 62387 (IEC, 2012) and ISO 12794 (ISO, 2000). In practice, the IEC 62387 standard is more exhaustive and hence should be preferred (Bordy et al., 2011).

\section{Design of a dosimeter dedicated to the measurement of $H_{\mathrm{p}}(3)$}

For the design of dosimeters, the ideal solution would be that the detector and its environment reproduce exactly the same conditions as defined for the operational quantity to be measured. Thus, when measuring $H_{\mathrm{p}}(3)$ or $H^{\prime}(3)$, the tissueequivalent material at the front of the detector should have a thickness of $3 \mathrm{~mm}$; a bolus of hemispherical or similar shape seems to be the most adapted (Bilski et al., 2011). Also, the thickness of the tissue-equivalent material behind the detector should be sufficient to reproduce the backscatter of the radiation if the dosimeter is not worn next to the skin. If the two previous conditions can be quite easily fulfilled, the composition of the detector itself is more problematic because it is not strictly equivalent to tissue in most cases. This composition and thus the variation of the response according to energy is an intrinsic characteristic of the detector. If this variation brings the type test results outside the criteria, a case needs to be added around the detector. Its composition and/or its shape including the front and rear faces must be adapted so that the criteria for energy and angle responses are fulfilled. Today, passive dosimeters are mainly based on a $\mathrm{LiF}$ thermo-luminescent detector, whereas for active dosimeters, silicon detectors are almost exclusively used. It is, however, necessary to mention that the response of active dosimeters in pulsed radiation fields with very high instantaneous dose rates decreases with the dose rate in most cases (Denoziere et al., 2007; Clairand et al., 2008).

\footnotetext{
5 www.meditest.fr
}

\section{Wearing conditions of a dosimeter designed for measuring $H_{\mathrm{p}}(3)$}

A dosimeter specifically designed to measure $H_{\mathrm{p}}(3)$ must be worn at the level of the eye. The dosimeter must be worn so that it cannot underestimate the exposure of the lens. It can be worn to the left or to the right or between the eyes; this has not been specified up to now. Wearing the dosimeter between the eyes would allow, to some extent, an average estimate of the exposure of the two lenses, whereas wearing the dosimeter on the right or on the left is better because it allows measurement of the maximum value of the exposure. If small detectors are used, three detectors can be inserted into a headband. If individual protection is used, for example glasses, the dosimeter must be put under this protection to give information about the real exposure to the lens. If the detector is put on the glasses, corrections must be applied to the results. As this correction depends on the energy of the radiation, the correction must be adapted to the radiation quality; that is to say, the radiation field must be known. A lot of models of glasses are commercially available; one should choose those with lateral and central shields to avoid lateral irradiation, similar to the case of the lack of underside protection for faceguards.

The choice of the dosimeters presented hereafter is quite arbitrary and not exhaustive. It gives an idea of the dosimeter characteristics that can be achieved (see the individual websites to update this data and see pictures of the dosimeters). In most of the models, a headband is used to maintain the dosimeter(s). It allows a morphological adaptation that is as ergonomic as possible. The dosimeter EYE-D ${ }^{\mathrm{TM}}$ (Radcard) was developed within the framework of the project ORAMED and was tested by the LNHB. It is based on a MCP$\mathrm{N}(\mathrm{LiF}: \mathrm{Mg}, \mathrm{Cu}, \mathrm{P})$ detector ${ }^{6}$. The photon energy dependency is within $+/-20 \%$ between $30 \mathrm{keV}$ and $1.33 \mathrm{MeV}$. The dose range is $10 \mu \mathrm{Sv}$ to $10 \mathrm{~Sv}$. The angular response is within $20 \%$ between 0 and $80^{\circ}$ (cylindrical phantom). The dosimeter proposed by the UK company Rotunda Scientific Technologies is based on one or several TLD Harshaw "chipstrates" covered by a 3 -mm-thick PTFE filter ${ }^{7}$. A similar design is proposed by the Health Protection Agency (HPA) but with a 1.5-mm-thick PTFE filter and a TLD Harshaw EXTRADTM ${ }^{8}$. The headband version has a claimed dose range from $0.15 \mathrm{mSv}$ to $10 \mathrm{~Sv}$; an energy range for photons from $16 \mathrm{keV}$ to $0.662 \mathrm{MeV}$; and an angle of incidence range up to $45^{\circ}$. The DOSIRIS model proposed by the IRSN also uses a kind of rigid headband; it is based on a TLD $\left.{ }_{7} \mathrm{LiF}: \mathrm{Mg}, \mathrm{Ti}\right)$ detector covered by a $3-$ mm-thick polypropylene bolus. A dose range from $100 \mu \mathrm{Sv}$ to $50 \mathrm{~Sv}$ for both beta particles and photons is claimed for energy down to $20 \mathrm{keV}$ and $700 \mathrm{keV}$ for photons and beta particles, respectively ${ }^{9}$.

\footnotetext{
${ }^{6}$ Detailed information can be found at www.radpro-int.com/assets/ eye-d.pdf.

7 Details can be found at http://www.rotundascitech.com/ EyeDosimetry.html.

8 More details can be found at http://www.hpa.org.uk.

${ }^{9}$ More information can be found at http://dosimetre.irsn.fr/fr-fr/ Documents/Fiches\%20produits/IRSN_Fiche_dosimetre_Cristallin. pdf.
} 


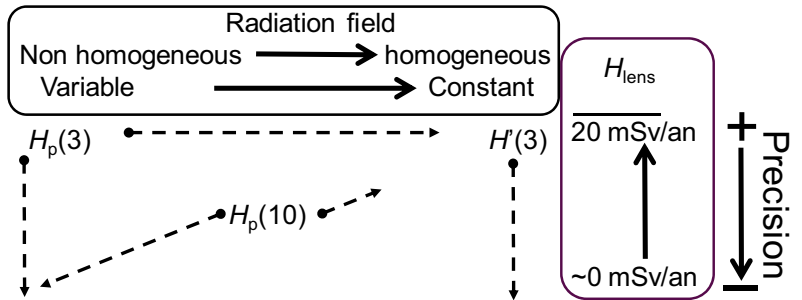

Figure 4. Possibilities for a direct measurement or an indirect evaluation of $H_{\mathrm{p}}(3)$ depending on the required precision and the homogeneity and constancy of the radiation field.

Landauer proposes a different design which can be fixed to glasses or a mask under the protective lead-loaded glass layer $^{10}$.

\section{Choice of the method for eye lens dose monitoring}

The first thing to do before choosing a method to monitor the doses is to carry out a workplace study. It allows the radiation field, in terms of direction and energy, to be characterized. It also allows the estimation of dose equivalent rates, on which the precision required for the measurement of the dose equivalents depends. Two possibilities are offered:

- the direct measurement of $H_{\mathrm{p}}(3)$ wearing a dosimeter close to the eye lens,

- or the indirect evaluation of $H_{\mathrm{p}}(3)$ through another dose equivalent quantity, for example in this paper $H_{\mathrm{p}}(10)$ measured on the trunk, and then applying a correction coefficient to derive $H_{\mathrm{p}}(3)$. One should keep in mind that any correction will lead to a degradation of the precision of the measurements.

Figure 4 illustrates the following statements: On one hand, if the annual limit is likely to be met, then the need in terms of precision is the greatest; therefore, the best choice is to wear a dosimeter allowing a direct measurement of $H_{\mathrm{p}}(3)$, especially in the case of inhomogeneous and varying exposure. One can mention the similarity between the dosimetry for extremities (finger, wrist and ankle) and for the lens; indeed, in both cases, the spatial and temporal homogeneity plays an essential role in the choice of the methods to evaluate skin and eye lens equivalent doses. So, if it can be inferred that it is necessary to use dedicated dosimeters to evaluate skin doses, in similar situations, it is likely that it could be inferred that it is also necessary to use a dedicated dosimeter to evaluate eye lens doses despite the constraints of wearing an additional dosimeter. This dedicated dosimeter is especially needed since the annual exposure limit for the eye lens has been drastically lowered. On the other hand, if the exposure is homogeneous and constant, area dosimeters calibrated and type-tested in terms of $H^{\prime}(3)$ can be used. Of course, the homogeneity and the constancy must be demonstrated during the period of use of the dosimeters for the workplaces concerned.

\footnotetext{
${ }^{10} \mathrm{http}: / /$ www.landauer-fr.com/lentreprise/actualites.html.
}

In all other cases, for varying levels of dose and homogeneity, according to the precision requirements, the results of whole-body dosimeters in terms of $H_{\mathrm{p}}(10)$ can be used to derive the eye lens doses. In such a case a correction factor has to be applied to the results to take into account the discrepancies between the quantities themselves and, if any, the gradients of dose highlighted during the workplace study between the eye and the breast. The first correction can be calculated by balancing the difference between the air kerma conversion coefficients and the dose equivalents according to the energy and angle of incidence of the radiation (see Figures 1 and 2 and Tables 1 and 2).

In this section we deal with the correction due to the nonhomogeneity of the radiation field between the locations of the dosimeters (second correction).

The knowledge of the spatial distribution of the radiation field allows the range of the gradients of the dose to be known between the various locations for dosimeters worn at the extremities, close to the eyes or on the chest. We can derive this homogeneity/inhomogeneity in terms of the ratio of dose equivalents normalized to the dose equivalent on the chest, $H_{\mathrm{p}}(10)$. Thus, one understands that wearing individual protection (apron, glasses and gloves) and the use of collective protection can be at the origin of a non-homogeneous radiation field and that considering staff movements, a perfect homogeneity of the radiation field is very unlikely. The highest values of $\mathrm{R}$ correspond to the most inhomogeneous radiation field. By defining $\mathrm{R}=H_{\mathrm{p}}(3) / H_{\mathrm{p}}(10)$, then $H_{\mathrm{p}}(3)$ can be derived from the $H_{\mathrm{p}}(10)$ measurements.

It is of interest to define a maximum value of $H_{\mathrm{p}}(10)$, $H_{\mathrm{p}}(10)_{\max }$, below which there is no risk of deriving a $H_{\mathrm{p}}(3)$ value below the limit while the real value of $H_{\mathrm{p}}(3)$ exceeds this limit.

Taking into account the limit of exposure for $H_{\mathrm{p}}(3)$, $20 \mathrm{mSv}$ on average over 5 years or $50 \mathrm{mSv}$ over one year ${ }^{11}$, one can substitute $H_{\mathrm{p}}(3)$ for these exposure limits to define a maximum value of $H_{\mathrm{p}}(10)$ such as :

$$
H_{\mathrm{p}}(10)_{\max 20}=20 / \mathrm{R} \quad \text { or } \quad H_{\mathrm{p}}(10)_{\max 50}=50 / \mathrm{R} .
$$

Thus, for example, if the ratio $\mathrm{R}$ is equal to five, it means that at this workplace, the limit of exposure in terms of $H_{\mathrm{p}}(3)$ corresponds to a maximum value of $H_{\mathrm{p}}(10)$ of either $4 \mathrm{mSv}$ or $10 \mathrm{mSv}$, depending on whether the limit of $20 \mathrm{mSv}$ or $50 \mathrm{mSv}$ is applied. Over these measured values of $H_{\mathrm{p}}(10)$, the limit in terms of $H_{\mathrm{p}}(3)$ is exceeded.

Considering the extended uncertainty $U\left(H_{\mathrm{p}} 10\right)$ on the measurement of $H_{\mathrm{p}}(10)$, one can change equation 1 as:

$$
\begin{aligned}
& H_{\mathrm{p}}(10)_{\max 20}+U\left(H_{\mathrm{p}} 10\right)=20 / \mathrm{R} \\
& \text { and } H_{\mathrm{p}}(10)_{\max 20}=(20 / \mathrm{R})-U\left(H_{\mathrm{p}} 10\right), \\
& H_{\mathrm{p}}(10)_{\max 50}+U\left(H_{\mathrm{p}} 10\right)=50 / \mathrm{R} \\
& \text { and } H_{\mathrm{p}}(10)_{\max 50}=(50 / \mathrm{R})-U\left(H_{\mathrm{p}} 10\right) .
\end{aligned}
$$

Thus, one can be sure at the $95 \%$ or $99.8 \%$ or $99.99 \%$ confidence level, depending on the coverage factor required, that

\footnotetext{
11 For France the option is still not decided.
} 
Table 3. Values of $H_{\mathrm{p}}(10)_{\max 20}$ and $\mathrm{R}$ calculated with equation (4) for a $H_{\mathrm{p}}(3)$ limit equal to $20 \mathrm{mSv}$ and $H_{0}=0.17 \mathrm{mSv}$.

\begin{tabular}{lccc}
\hline $\boldsymbol{H}_{\mathrm{p}}(\mathbf{1 0})_{\max 20}(\mathbf{m S v})$ & $\mathrm{U}$ \% & $\mathbf{U}(\mathbf{m S v})$ & $\mathbf{R}$ \\
\hline 0.1 & 100 & 0.1 & 100 \\
1 & 70 & 0.7 & 11.8 \\
6 & 50 & 3 & 2.16 \\
10 & 50 & 5 & 1.31 \\
20 & 50 & 10 & 0.66 \\
\hline
\end{tabular}

the limit of exposure in terms of $H_{\mathrm{p}}(3)$ is not exceeded. If $\mathrm{U}=$ $2 \mathrm{mSv}$ and $\mathrm{R}=5$, the limit of exposure in terms of $H_{\mathrm{p}}(3)$ corresponds to a value of $H_{\mathrm{p}}(10)_{\max 20}$ equal to $2 \mathrm{mSv}$ and $H_{\mathrm{p}}(10)_{\max 50}$ equal to $8 \mathrm{mSv}$ if the limit is $20 \mathrm{mSv}$ or $50 \mathrm{mSv}$, respectively. Above these measured values of $H_{\mathrm{p}}(10)$, the limit in terms of $H_{\mathrm{p}}(3)$ is exceeded.

The trumpet curves (ISO 14146, 2000) are used to derive the maximum error allowed depending on measured values of $H_{\mathrm{p}}(10)$. The upper limit is given by

$$
\begin{aligned}
& H_{\mathrm{p}}(10)_{\mathrm{ul}}= 1.5 H_{\mathrm{p}}(10)_{\mathrm{t}}\left(1+H_{0} /\left(2 H_{0}+H_{\mathrm{p}}(10)_{\mathrm{t}}\right)\right) \\
& \text { if } H_{\mathrm{p}}(10)_{\mathrm{t}} \geqslant H_{0} \\
& \text { and } H_{\mathrm{p}}(10)_{\mathrm{ul}}= 2 H_{\mathrm{p}}(10)_{\mathrm{t}} \\
& \text { if } H_{\mathrm{p}}\left(10_{\mathrm{t}}<H_{0} .\right.
\end{aligned}
$$

The lower limit is given by

$$
\begin{aligned}
H_{\mathrm{p}}(10)_{11} & =H_{\mathrm{p}}(10)_{\mathrm{t}} / 1.5\left(1-2 H_{0} /\left(2 H_{0}+H_{\mathrm{p}}(10)_{\mathrm{t}}\right)\right) \\
& \text { if } H_{\mathrm{p}}(10)_{\mathrm{t}} \geqslant H_{0} \\
\text { and } H_{\mathrm{p}}(10)_{11}= & 0 \quad \text { if } H_{\mathrm{p}}(10)_{\mathrm{t}}<H_{0}
\end{aligned}
$$

where $H_{\mathrm{p}}(10)_{\mathrm{t}}$ is the true value of the dose equivalent. $H_{0}$ is the lowest dose equivalent required to be measured; here $0.17 \mathrm{mSv}$ for $H_{\mathrm{p}}(10)$ for photons for a monthly wearing period and a limit equal to $20 \mathrm{mSv}$ (European Commission,1994); a value of $0.41 \mathrm{mSv}$ could be used for a limit equal to $50 \mathrm{mSv} . H_{\mathrm{p}}(10)_{\mathrm{ul}}$ and $H_{\mathrm{p}}(10)_{11}$ are the upper and lower limits, respectively. It turns out that the upper limit is the most penalizing, therefore $H_{\mathrm{p}}(10)_{\mathrm{ul}}$ was taken into account to substitute $U\left(H_{\mathrm{p}} 10\right)$ for the maximum error in equation (2):

$$
\begin{aligned}
H_{\mathrm{p}}(10)_{\max 20}= & (20 / \mathrm{R})-H_{\mathrm{p}}(10) \\
& \times 1.5\left(1+H_{0} /\left(2 H_{0}+H_{\mathrm{p}}(10)\right)\right) \\
& \text { if } H_{\mathrm{p}}(10) \geqslant H_{0} \\
\text { and } H_{\mathrm{p}}(10)_{\max 20}= & (20 / \mathrm{R})-H_{\mathrm{p}}(10) \times 2 \\
& \text { if } H_{\mathrm{p}}(10)<H_{0} \\
\text { or } H_{\mathrm{p}}(10)_{\max 50}= & (50 / \mathrm{R})-H_{\mathrm{p}}(10) \\
& \times 1.5\left(1+H_{0} /\left(2 H_{0}+H_{\mathrm{p}}(10)\right)\right) \\
& \text { if } H_{\mathrm{p}}(10) \geqslant H_{0} \\
\text { and } H_{\mathrm{p}}(10)_{\max 50}= & (50 / \mathrm{R})-H_{\mathrm{p}}(10) \times 2 \\
& \text { if } H_{\mathrm{p}}(10)<H_{0} .
\end{aligned}
$$

Tables 3 and 4 give a few values taking into account the maximum error for a monthly period. It should be noted that a calculation using the uncertainty data of the RP 160 report (European Commission, 2009) gives very similar results.
Table 4. Values of $H_{\mathrm{p}}(10)_{\max 50}$ and $\mathrm{R}$ calculated with equation (4) for a $H_{\mathrm{p}}(3)$ limit equal to $50 \mathrm{mSv}$ and $H_{0}=0.17 \mathrm{mSv}$ and $0.41 \mathrm{mSv}$.

\begin{tabular}{lcccc}
\hline $\begin{array}{l}\boldsymbol{H}_{\mathrm{p}}(\mathbf{1 0})_{\max 50} \\
(\mathbf{m S v})\end{array}$ & $\mathbf{U}$ \% & $\mathbf{U}(\mathbf{m S v})$ & $\begin{array}{c}\mathbf{R} \\
\left(\boldsymbol{H}_{\mathbf{0}}=\mathbf{0 . 1 7} \mathbf{~} \mathbf{m S v}\right)\end{array}$ & $\begin{array}{c}\mathbf{R} \\
\left.\boldsymbol{H}_{\mathbf{0}}=\mathbf{0 . 4 1} \mathbf{~} \mathbf{S v}\right)\end{array}$ \\
\hline 0.1 & 100 & 0.1 & 250 & 250 \\
1 & 70 & 0.7 & 29.6 & 27.2 \\
6 & 50 & 3 & 5.41 & 5.24 \\
10 & 50 & 5 & 3.28 & 3.21 \\
20 & 50 & 10 & 1.65 & 1.63 \\
50 & 50 & 25 & 0.66 & 0.66 \\
\hline
\end{tabular}

Of course, if $\mathrm{R}$ is very large, it should only be applied to very low values of $H_{\mathrm{p}}(10)$ to derive $H_{\mathrm{p}}(3)$. This case would mean that the eye lens is the main organ to be irradiated, whereas the rest of the body is not significantly irradiated. This can be the case, for example, if a lead apron is worn but no eye protection.

During the workplace study an uncertainty, $U(R)$, is associated with the measurement of $R$. It is advisable to use $R+U(R)$ instead of $\mathrm{R}$ to take into account a ratio value which will not underestimate $H_{\mathrm{p}}(3)$. Thus, one sets up a relationship between the inhomogeneity of the radiation field and the need for a direct measurement of $H_{\mathrm{p}}(3)$, the latter being evaluated on the basis of a measurement of $H_{\mathrm{p}}(10)$.

$$
\begin{aligned}
H_{\mathrm{p}}(10)_{\max 20}= & (20 /(\mathrm{R}+\mathrm{U}(\mathrm{R})))-H_{\mathrm{p}}(10) \\
& \times 1.5\left(1+H_{0} /\left(2 H_{0}+H_{\mathrm{p}}(10)\right)\right) \\
& \text { if } H_{\mathrm{p}}(10) \geqslant H_{0} \\
\text { and } H_{\mathrm{p}}(10)_{\max 20}= & (20 /(\mathrm{R}+\mathrm{U}(\mathrm{R})))-H_{\mathrm{p}}(10) \times 2 \\
& \text { if } H_{\mathrm{p}}(10)<H_{0} \\
\text { or } H_{\mathrm{p}}(10)_{\max 50}= & \left(50 /(\mathrm{R}+(\mathrm{U}(\mathrm{R})))-H_{\mathrm{p}}(10)\right. \\
& \times 1.5\left(1+H_{0} /\left(2 H_{0}+H_{\mathrm{p}}(10)\right)\right) \\
& \text { if } H_{\mathrm{p}}(10) \geqslant H_{0} \\
\text { and } H_{\mathrm{p}}(10)_{\max 50}= & (50 /(\mathrm{R}+\mathrm{U}(\mathrm{R})))-H_{\mathrm{p}}(10) \times 2 \\
& \text { if } H_{\mathrm{p}}(10)<H_{0} .
\end{aligned}
$$

For example, for a value of $\mathrm{R}=4$ the value of $H_{\mathrm{p}}(10)_{\max }$ is approximately equal to $3.1 \mathrm{mSv}$ and $7.9 \mathrm{mSv}$, respectively, depending on which exposure limit is used (Figure 5).

The results of the workplace study can be plotted on a twodimensional graph. The $\mathrm{x}$-axis represents the ratio of the doses (and hence the inhomogeneity) and the $\mathrm{y}$-axis represents the dose. One can plot the values of $\mathrm{R}+\mathrm{U}(\mathrm{R})$ and $H_{\mathrm{p}}(10)_{\max }$ on this graph and then visualize the domain in which a direct measurement must be used or an indirect evaluation of $H_{\mathrm{p}}(3)$ can be used.

\section{Conclusion}

New epidemiological studies, followed by the results of ORAMED, the recommendation of the ICRP, the new European directive 2013/59 (EURATOM, 2013), and the ISO and IEC standards published (or to be published), have already significantly changed the process of the occupational dosimetry 


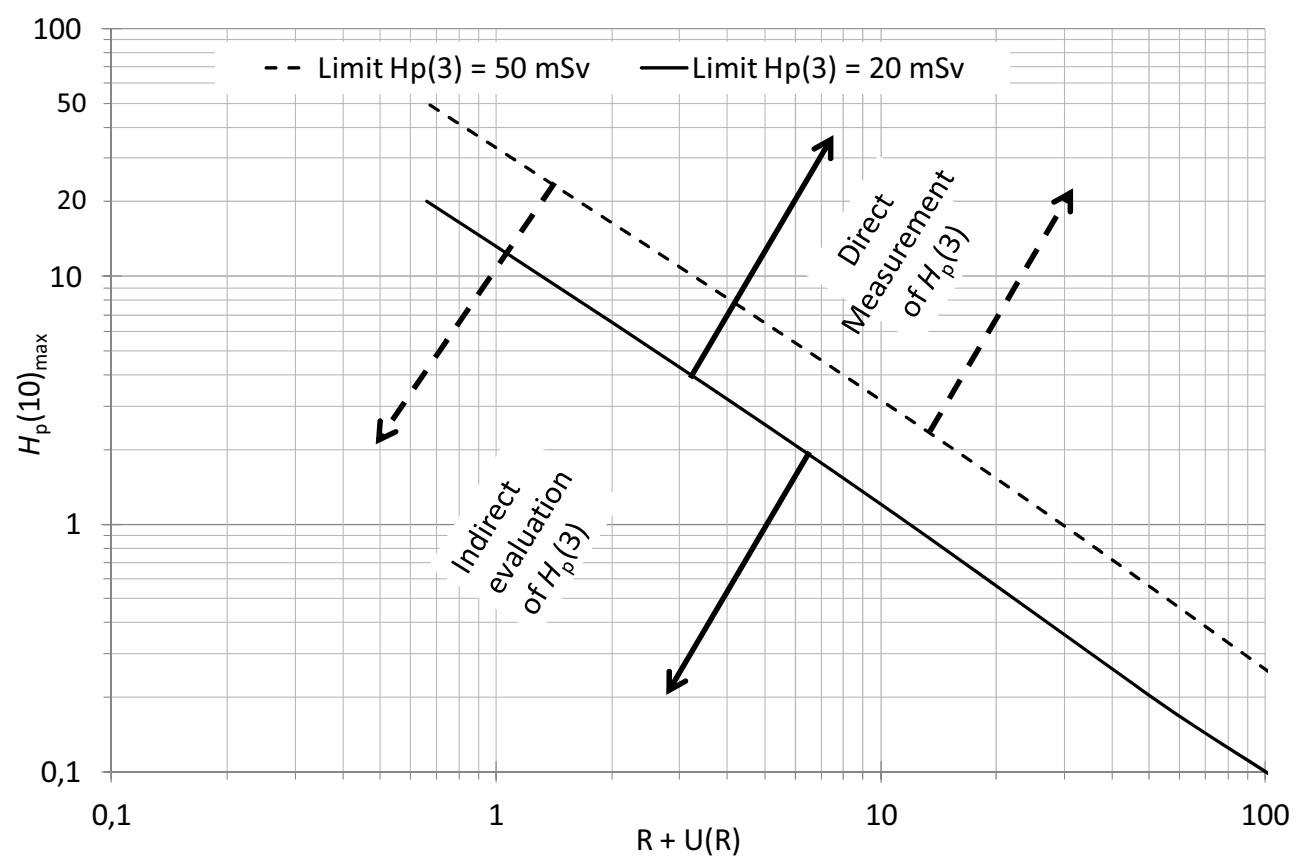

Figure 5. Visualization of the domains for a direct measurement or indirect evaluation of $H_{\mathrm{p}}(3)$.

of the eye lens for radiation protection. It is no longer possible to rely exclusively on the results of whole-body dosimeters to estimate the eye lens exposure. A compromise solution between the constraint of wearing an additional dosimeter close to the eye, and the need to monitor the lens exposure, is needed. This paper proposes an objective index to decide when an eye lens dosimeter should be worn and when it might be possible to evaluate $H_{\mathrm{p}}(3)$ from the whole-body dosimeter. The same method can be applied to define a domain for the evaluation of $H_{\mathrm{p}}(10)$ through $H^{*}(10)$ or other dose equivalent quantities or the evaluation of $H_{\mathrm{p}}(0.07)$ through $H^{\prime}(0.07)$; the main difficulty is choosing a reliable value of $\mathrm{R}$ based on a study of the particular workplace.

Acknowledgements. The author wishes to thank Mark Kellett.

\section{References}

Bilski P. et al. (2011) The new eye- ${ }^{\mathrm{TM}}$ dosemeter for measurements of $H_{\mathrm{p}}(3)$ for medical staff, Radiat. Meas. 46 (11), 1239-1242.

Bordy J.M. et al. (2011) Proposals for the type tests and calibration methodology of passive eye lens dosimeters in interventional cardiology and radiology workplaces, Radiat. Meas. 46 (11), 1235-1238.

Chodick G. et al. (2008) Risk of cataract after exposure to low doses of ionizing radiation: a 20 -year prospective cohort study among US radiologic technologists, Am. J. Epidemiol. 168, 620-31.

Clairand I. et al. (2008) Intercomparison of active personal dosemeters in interventional radiology, Radiat. Prot. Dosim. 129 (1-3), 340-345.

Daures J. et al. (2009) Conversion coefficients from air kerma to personal dose equivalent $H_{\mathrm{p}}(3)$ for eye-lens dosimetry, ISSN-04293460, CEA-R-6235 Saclay, France.
Daures J. et al. (2011) Monte Carlo determination of the conversion coefficients $H_{\mathrm{p}}(3) / K_{\mathrm{a}}$ in a right cylinder phantom with penelope code. Comparison with "mcnp" simulations, Radiat. Prot. Dosim. 144 (1-4), 37-42.

Denoziere M. et al. (2007) Pulsed X-rays for interventional radiology: tests on active personal dosemeters (APD) (European project FP7 ORAMED WP3), CEA-R-6233, ISSN-0429-3460.

Dusciac D. et al. (2013) Étude pour l'établissement d'une référence de faisceaux photoniques de haute énergie pour la radioprotection. In: Proceedings of Congrès National de radioprotection, SFRP, 11-13 juin 2013, Bordeaux. http://www.nucleide.org/ Publications/2013.htm.

EURATOM (2013) European directive 2013/59. Basic Safety Standards for protection against the dangers arising from exposure to ionizing radiation.

European Commission PR 73 (1994) Technical Recommendations for Monitoring Individuals Exposed to External Radiation, report EUR 14852 EN.

European Commission PR 160 (2009) Technical Recommendations for Monitoring Individuals Exposed to External Radiation, Radiation Protection No 160.

Gualdrini G. et al. (2011) A new cylindrical phantom for eye lens dosimetry development, Radiat. Meas. 46, 1231-1234.

Gualdrini G. et al. (2013) Air kerma to $H_{\mathrm{p}}(3)$ conversion coefficients for photons from $10 \mathrm{keV}$ to $10 \mathrm{MeV}$, calculated in a cylindrical phantom, Radiat. Prot. Dosim. 154 (4), 517-521.

ICRP publication 74 (1995) Conversion coefficients for use in radiological protection against external radiation, ICRP.

ICRP publication 116 (2010) Conversion coefficients for radiological protection quantities for external radiation exposures, ICRP.

ICRP (2011) International Commission on Radiological Protection Statement on tissue reactions, ICRP ref 4825-3093-1464.

ICRU report 43 (1988) Determination of dose equivalents from external radiation sources- Part 2, Bethesda. 
ICRU report 47 (1992) Measurement of dose equivalent from external photons and electron radiations, International commission on radiation units and measurements, Bethesda.

ICRU report 57 (1995) Conversion coefficients for use in radiological protection against external radiation, ICRU, Bethesda.

IEC 61526 (2005) Radiation protection instrumentation Measurement of personal dose equivalents $H_{\mathrm{p}}(10)$ and $H_{\mathrm{p}}(0,07)$ for $\mathrm{X}$, gamma, neutron and beta radiations - Direct reading personal dose equivalent meters and monitors.

IEC 61066 (2006) Thermo luminescence dosimetry systems for personal and environmental monitoring.

IEC 60846 (2007) Radiation protection instrumentation - Ambient and/or directional dose equivalent (rate) meters and/or monitors for beta, $\mathrm{X}$ and gamma radiation - Part 2: High range beta and photon dose and dose rate portable instruments for emergency radiation protection purposes.

IEC 62387 (2012) Radiation protection instrumentation - Passive integrating dosimetry systems for environmental and personal monitoring.

ISO 4037 (1999) X and gamma reference radiation for calibrating dosemeters and doserate meters and for determining their response as a function of photon energy- Part 3: Calibration of area and personal dosemeters and the measurement of their response as a function of energy and angle of incidence.

ISO 12794 (2000) Individual thermo luminescence dosemeters for extremities and eyes, ISO, Geneva.

ISO 14146 (2000) Criteria and performance limits for the periodic evaluation of processors of personal dosemeters for $\mathrm{X}$ and gamma radiation. ISO, Geneva.

ISO 29661 (2012) Reference radiation fields for radiation protection Definitions and fundamental concepts, ISO, Geneva.

Mariotti F. et al. (2009) ORAMED project eye-Lens dosimetry, a new Monte Carlo approach to define the operation quantity $H_{p}(3)$, ISSN/0393-3016, RT/2009/1/BAS ENEA Rome.

Nakashima E. et al. (2006) A reanalysis of atomic-bomb cataract data, 2000-2002: a threshold analysis, Health Phys. 90, 154-60.

Pelowitz D.B. (2005) MCNPX ${ }^{\mathrm{TM}}$ User's Manual Version 2.5.0. Los Alamos National Laboratory report LA-CP-05-036.

Salvat F. et al. (2006) "PENELOPE-2006. A Code System for Monte Carlo Simulation of Electron and Photon Transport", OECD/NEA.

Worgul B.V. et al. (2007) Cataracts among Chernobyl Clean-up Workers: Implications Regarding Permissible Eye Exposure., Radiat. Res. 167, 233-243.

Cite this article as: J.-M. Bordy. Monitoring of eye lens doses in radiation protection. Radioprotection 50(3), 177-185 (2015). 